\title{
Acquiring and Exploiting the User's Knowledge in Guidance Interactions
}

\author{
Eyal Shifroni and Uzzi Ornan \\ Department of Computer Science \\ Technion - Israel Institute of Technology \\ Technion City, Haifa 32000, Israel \\ email: shifroni@cs.technion.ac.il, ornan@cs.technion.ac.il
}

January 5,1992

\section{Introduction}

This paper presents a model for Flexible Interactive Guidance System (FIGS) that provides people with instructions about natural tasks. The model is developed on the basis of a phenomenological analysis of human guidance and illustrated by a system that gives directions in geographical domains. The instructions are provided through a dialog adapted both in form and content to user's needs. The main problem addressed is how to provide a user-adapted guidance during the normal course of the guidance dialog, without introducing a special time consuming sub-dialog to gain information about the user's state of knowledge.

A user-adapted guidance system must collect information about the user's knowledge of the guidance domain and build a User Model (UM). It is known that a UM can improve the behavior of dia$\log$ systems and contribute to the ease of their usage and the naturalness of their response ((Rich, 1979)). However, a UM may also have negative effects on the system's behavior, since the process of its acquisition may increase both the time and the effort the user must invest in the interaction. The model suggested here addresses this problem by weighing the effort required to acquire the UM against the benefits of its usage.

\section{Background}

User modeling systems that were built during the 80 s were developed for advisory systems. Early systems used an explicit method to acquire the UM (e.g., (Rich, 1979)). In such a method facts about the user are provided to the UM by the user himself. This method however, has a major drawback, since the user who is interested in obtaining information from the system, is forced to provide answers to a sequence of questions posed by the system. Recently, several methods to infer facts about the user implicitly were suggested (see (Kass, 1990) for an overview). These methods exploit the user's questions and the user's answers to questions posed by the system, to indirectly infer new facts about him, and are thus able to construct a detailed UM based on a relatively short dialog.

The explicit acquisition method was described in the recent literature as inferior to the implicit method because of its consumption of the user's time. However, implicit acquisition includes a risk of missing essential facts about the user, and hence may cause the system to provide unnecessary information that is already known to the user.

\section{Integrating Explicit and Im- plicit Methods}

The model suggested here integrates the explicit acquisition method with a variety of implicit methods in a context of a Guidance dialog. In contrast to advisory dialogs that usually contain an interview phase aiming to elicit from the user information required for the advice generation, in guidance interactions most of the dialog consists of instructions provided by the system, and user's responses to these instructions. Although questions to the guidance recipient - the guidee - do occur, they do not constitute a separate phase but are rather scattered in the instruction sequence. In the current study and 
in other studies of human guidance dialogs it was found that in such dialogs any instruction made by the guide is followed by a guidee's response such as question, interruption, or confirmation-signal. In human interaction confirmation-signals are provided by either verbal (e.g., "ahm") or non-verbal (e.g., head nodding) means, this can be simulated in humancomputer interaction by a special "continue" button that is used to signal the system to move to the next instruction.

We claim that any instruction-response pair in the guidance sequence can serve as a basis for inferences about the guidee. In general, each such pair type triggers different inferences and consumes a different amount of the user's resources (time, effort, patience). For example, inferences can be drawn from an instruction/confirmation-signal pair; the confirmation signal shows that the user understands the instruction and thus affirms the assumptions which led to its production. Such default inferences are less certain than those based on answers to explicit questions, but they do not consume the resources of the guidee: No special action from him is required; in fact the guidee may not even be aware to the fact that inferences about him are being made. A strategy of explicit questioning is justified only when the needed fact is informative, and there was no way in which the system could infer it from the previous dialog. In any other case a more implicit strategy should be preferred.

This view extends the explicit-implicit distinction mentioned above since each utterance pair type facilitates a different acquisition method, and each such method has a different level of explicitness. Our model provides therefore, a new definition of the notion of explicitness which extends the previous notion in two ways. First, we distinguish between $A c$ quisition Explicitness and Transmission Explicitness, this way the notion can be applied both for information flowing from guide to guidee (guidance information), and for information flowing from guidee to guide (user modeling information). Second, we allow various levels of explicitness, rather than the two extremes only. The system's explicitness level is defined in terms of the interactive effort that is invested in the dialog. This can either be the effort required to provide a fact to the guidee, or the effort required to acquire a fact about him.

We define FIGS as a system that provides interactive, user-adapted guidance, and satisfies the following two conditions: (1) It is equipped with a set of instruction-acquisition strategies, each of which is characterized by a different level of acquisition ex- plicitness and transmission explicitness; and (2) It has a mechanism to dynamically select a strategy. This mechanism attempts to reach optimality with respect to the interactive effort required for providing the information needed by the guidee.

The suggested model implements the foregoing considerations, it includes four instructionacquisition strategies: (1) Question - the user provides a needed fact, (2) Explanation - no facts about the user are used, (3) Explicit Assumption - the instruction is based on an assumption about the user, and the assumption is mentioned, and (4) Implicit Assumption - an assumption is used without mentioning it.

A major feature of our model is that UM acquisition considerations are integrated into the FIGS's utterance planning process. Hence, the user modeling and the instruction generation are done incrementally by inter-related processes. We use heuristic rules to select among the four strategies; these rules weigh four discourse parameters: information content, user knowledge (as described by the current UM), likelihood to acquire new facts about the user, and consumption of user's resources.

The computational model we suggested was implemented by a computer program (called FIGS1) that offers directional instructions in a complex university building. FIGS1 uses heuristic rules to control both the strategy selection and the UM construction. A more detailed exposition of our model and its implementation can be found in (Shifroni \& Shanon, 1991), and (Shifroni \& Ornan, 1991).

\section{References}

Kass, R. (1990). Building a user model explicitly from a cooperative advisory dialog. In Second International Workshop on User Modeling.

Rich, E. (1979). User modeling via stereotypes. Cognitive Science, 3(4), 329-354.

Shifroni, E. \& Ornan, U. (1991). FIGS1: A flexible guidance dialog system. In Proceedings of the 8th Israeli Symposium on Artificial Intelligence, (pp. 51-68). Color print press Jerusalem.

Shifroni, E. \& Shanon, B. (1991). Interactive user modeling: An integrating explicit-implicit approach. Submitted to the journal of User Modeling and User Adapted Interaction. 